\title{
CAPP SYSTEM PROCESS SEQUENCE OPTIMIZATION BASED ON GENETIC ALGORITHMS
}

\author{
Shen Lei ${ }^{1}$, Yigang $\mathrm{Hu}^{2}$, Cheng Jiang ${ }^{3}$, Liu Yuan ${ }^{4}$ \\ ${ }^{1}$ Department of Mechanical Engineering, Shanghai University of Engineering Science, Shanghai 201600 China \\ ${ }^{2}$ Department of Mechanical Engineering, Shanghai University of Engineering Science, Shanghai 201600 China \\ ${ }^{3}$ Department of Mechanical Engineering, Shanghai University of Engineering Science, Shanghai 201600 China \\ ${ }^{4}$ Department of Mechanical Engineering, Shanghai University of Engineering Science, Shanghai 201600 China
}

\begin{abstract}
The arrangement of the process sequence is one of the key technologies in CAPP system antomatically generating process diagram, is the key whether the process design is reasonable or not, is also one of the difficulties in process design. To solve this problem, this paper proposes using genetic algorithms to optimize process sequence based on process constraints and uses a housing part to vertify.The result shows that using genetic algorithms can effectively optimize the process routes and get the optimal or near-optimal routings which meet the production requirements.
\end{abstract}

Keywords: Process sequence, Genetic algorithms, CAPP, Process diagram

$* * *$

\section{INTRODUCTION}

In the process of CAPP system automatically generating process diagram, process sequence decision-making is an extremely complex process, including selection from a variety of process schemes and the sorting of processing method. For machining complex parts which have many features, there are many constraints between features, type complex, and there are many contradictions between them. Using traditional ways will face a lot of difficulties and resistance. Therefore, this paper choose a routing decision algorithm: the genetic algorithm based on process constraints. This decision method utilizes the features of global search in genetic algorithms. First of all, in accordance with certain principles of sequencing technology, sort the processing methods of parts surface features according to certain sequence sorting, then determine the processing sequence of parts and optimize the process sequence.

\section{ESTABLISH THE MATHEMATICAL MODEL OF THE PROCESS SEQUENCE}

The sorting of process sequence, after defining all process characteristics and processing methods of parts, is a process that find out the optimal or near-optimal process route from the machining chain have been identified.

Assume parts processing chain identified is composed by $n$ machining feature units(f1, $f 2, \ldots, f n)$, then the set composed by all feature processing units of part is:

$$
F=\left\{f_{1}, f_{2}, \ldots, f_{n}\right\}
$$

Where the processing sequence of $\mathrm{F}$ is random,rather than the optimum sequence. In the process of sorting process, $\mathrm{F}$ also must meet the corresponding conditions for machining parts.Here the constraints set as:

$$
C_{R}=\left\{c_{1}, c_{2}, \ldots, c_{m}\right\}
$$

Here, the process sequence that meet the constraints $\mathrm{CR}$ is denoted by $\mathrm{U}$, feature machining unit set having the biggest fitness which use the genetic algorithm to find is the optimum process sequence, that is:

$$
G_{s}=\left\{f_{s 1}, f_{s 2}, \ldots, f_{s n}\right\}
$$

\section{PRECEDENCE RELATION CONSTRAINT}

\section{SET OF THE PROCESSING PRECEDENCE}

There are some mandatory constraints and optimality constraints between the processing characteristics of a complex parts. The process of process sequence optimization in the CAPP system is first find the process sequence set meeting the mandatory constraints, then judge according to the standard of the optimal constraints,and finally get the processing sequence having the best quality. Here mandatory constraint set is denoted as $C_{\mathrm{a}}$ the optimality constraint set is denoted as $C_{\mathrm{b}}$.

Mandaory constraint set $\mathrm{Ca}$ is the constraints that show the
sequence in the process of machining, for example, first
reference after the other, first surface after hole, first
primary after secondary, sequence that its features decide,
and so on. Considering these mandatory constraints, We cite
logic symbols to represent complex constraints, for example,
$f_{1}\left(f_{2} \wedge f_{3} \wedge \ldots \wedge f_{n}\right)$ means the machining of feature f1 must be
machined after the feature $\left\{f_{2} f_{3} \ldots f_{n}\right\}$ completed; $f 1$ ( $f_{2}$
$\left.\vee f_{3} \vee \ldots \vee f_{n}\right)$ means after any of $\left\{f_{1} f_{2} \ldots f_{n}\right\}$ completed, $f 1$
can start machining. 
The optimal constraint set $\mathrm{Cb}$ is generally considered from the point of the machining precision, time, cost, for example, minimize the number of changing knife, minimize the number of clamp, clustering constraint, and so on.

\section{PROCESS OPTIMIZATION USING GENETIC}

\section{ALORITHM}

GA is a optimization method of global probability search, uses the natural selection in the process of biological evolution to optimize, its basic operation process includes: code, production of original population, calculation of the fitness, seed selection, cross, variation. For this paper about the problem of CAPP system process route optimization, specific steps are as follows:

(1) first determine the gene coding method, and randomly generate initial population, then begin the process of evolution.

For the scheduling problem of the parts' feature process units, this paper use the natural number coding, and adopts forms of phenotype coding and genotype coding. The phenotype coding is used to directly reflect the processing sequence. Suppose the part processing chain PC(n) which has $\mathrm{n}$ machining feature is $\left(\mathrm{fm}_{1}, \mathrm{fm}_{2}, \ldots, \mathrm{fm}_{\mathrm{n}}\right)$, then the phenotype coding is:

$$
p c=\left(m_{1}, m_{2}, \ldots, m_{n}\right)
$$

Where $m_{1}, m_{2}, \ldots m_{n} \in\{1,2, \ldots, n\}$, and $m_{1} \neq m_{2} \neq \ldots \neq m_{n}$.

The genotype coding is used for the processing of genetic algorithm, it is expressed as:

$$
g c=\left(g c_{1}, g c_{2}, \ldots, g c_{n}\right)
$$

Where $g c_{1}, g c_{2}, \ldots, g c_{n} \in\{1,2, \ldots, n\}$.

The process of decoding is the process from the genotype coding $g c$ to the phenotype coding $p c$.Specific process is as follows:

for $(\mathrm{i}=0 ; \mathrm{i}<\mathrm{n} ; \mathrm{i}++)\{$

To obtain $N E X T(i)=\left(f_{i 1}, f_{i 2}, \ldots, f_{i y}\right)$, a total of $t$ elements;

Compute $b=\left[t \times g c_{i} / n\right]+1$;

$P_{c i}=i b$; that is, select the first $b$ elements of set $N E X T(i)$ \}

(2) Decode individual's genetic code into reasonable processing chain through decoding algorithm, determine the fitness of all individuala according to $\mathrm{Cb}$, where high fitness individuals have a higher probability of breeding.
When designing the fitness function, this paper mainly considers the clamping number, number of changeing knife and clustering. Here the fitness function is defined as:

$$
F=a_{1} \times \text { Score }_{1}+a_{2} \times \text { Score }_{2}+a_{3} \times \text { Score }_{3}
$$

Where $a_{1}, a_{2}, a_{3}$ is the weight of clamping, tool change, clustering. Score ${ }_{1}$, Score $_{2}$, Score $_{3}$ is the corresponding score.

Choose individual regeneration to crossover and mutation according to the crossover rate and mutation rate, after several generations of evolution, the individuals with the highest fitness in the final population is the best process sequence.

For the cross process, first suppose that the right part of two parent chains are invariant, then each of the parental generation find the codes that their own right code chain don't have from another parental generation, and fill according to the sequence,finally form two offspring, as is shown in Figure 1. For the process of variation, choose a number of bar code chain from a new group, then random transform the position of two codes, complete gene mutation, as is shown in Figure 2.
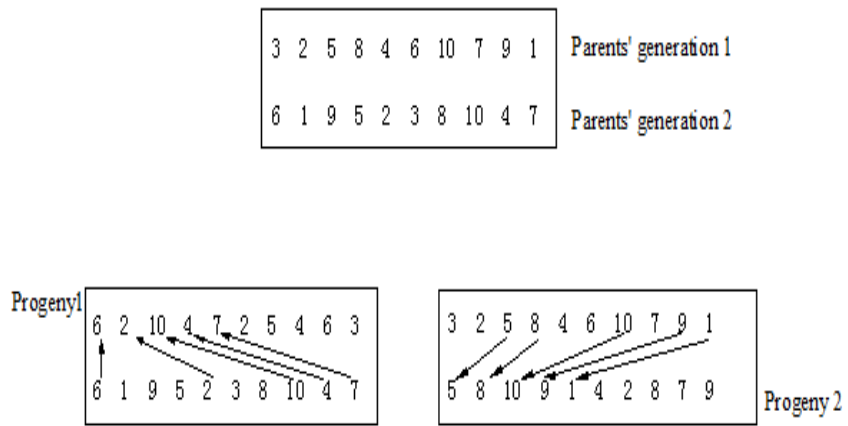

Fig-1: Cross process

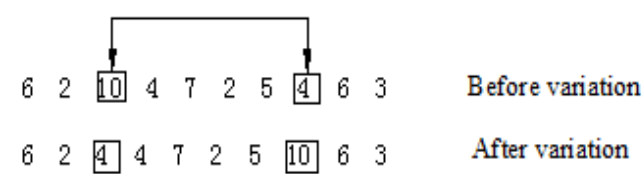

Fig-2: Variation process

After getting new group, calculate the fitness of individuals in the group, then select repeated and the process of crossover and variation, until the fitness of the group to achieve optimal,and then obtain the optimal process sequence.

\section{AN EXAMPLE OF CAPP PROCESS SEQUENCE OPTIMIZATION}

Taking the machining features of the part shown in Figure 3 for example, use genetic algorithms to optimize the process sequence. 


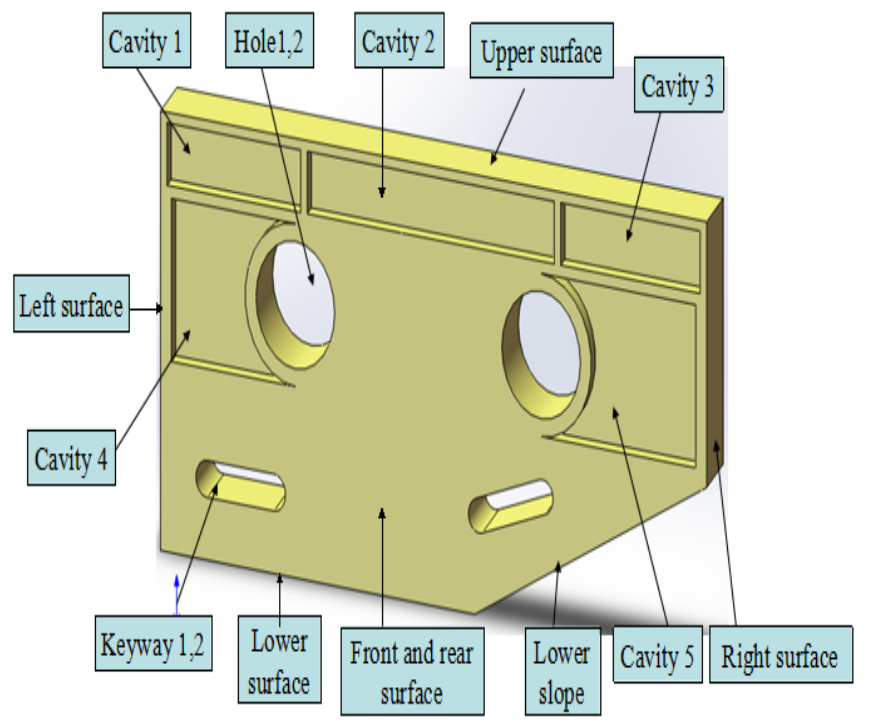

Fig-3: Example of part

The machining features of this part include: 6 planes; hole1,2; keyway1,2; and a total of 10 cavities which symmetrically distribute in the part. Through the knowledge of the process and the reasoning, we can get the processing methods of these features, as follows:

2 holes: $f_{1}\left(f_{4}\right)$ drill, $f_{2}\left(f_{5}\right)$ expend, $f_{3}\left(f_{6}\right)$ mill;

10 inner cavity surfaces: $f_{11}\left(f_{12}, \ldots, f_{20}\right)$ rough mill, $f_{21}$ $\left(f_{22}, \ldots, f_{30}\right)$ finish milling;

The upper and lower, left and right surfaces (surface 3,4,5,6): $f_{31}\left(f_{35}\right)$ rough mill, $f_{32}\left(f_{36}\right)$ finish-milling, $f_{33}\left(f_{37}\right)$ rough mill, $f_{34}\left(\mathrm{f}_{38}\right)$ finish-milling;

2 keyways: $f_{39}$ rough mill, $f_{40}$ finish-milling, $f_{41}$ rough mill, $f_{42}$ finish-milling;

Lower slope( surface 7): $f_{43}$ rough mill, $f_{44}$ finish-milling. Therefore, feature machining unit is:

$F=\left\{f_{1}, f_{2}, \ldots, f_{44}\right\}$

According to the analysis of the constraints, we can get the mandatory constraint set:

$$
C_{a}=\left\{\begin{array}{l}
\left(f_{7} \vee f_{9}\right)\left(f_{1} f_{2} \ldots f_{6} f_{8} f_{10} \ldots f_{44}\right),\left(f_{1} \vee f_{3}\right) \\
\left(f_{11} f_{12} \ldots f_{30} f_{39} \ldots f_{42}\right),\left(f_{8} \vee f_{10}\right)\left(f_{3} f_{6} f_{39} \ldots f_{42}\right)
\end{array}\right\}
$$

The optimization constraint set $C_{b}$ includes clamping type set $S U_{i}$, the tool set $T_{i}$ and the constraint set. Where the clamping type set is:

$$
\left\{S U_{1}, S U_{2}, S U_{3}\right\}=\left\{\begin{array}{l}
\left\{f_{9}, f_{10}, f_{21}, \ldots, f_{30}\right\},\left\{f_{35}, \ldots, f_{38}\right\}, \\
\left\{f_{1}, \ldots, f_{8}, f_{11}, \ldots, f_{20}, f_{39}, \ldots, f_{44}\right\}
\end{array}\right\}
$$

The tool set is:

$$
\left\{T_{1}, \ldots, T_{5}\right\}=\left\{\begin{array}{l}
\{\text { Rough milling process of } 10 \text { cavities }\}, \\
\{\text { Fine milling process of } 10 \text { cavities }\}, \\
\left\{f_{1}, f_{4}\right\}, \\
\left\{f_{2}, f_{3}, f_{5}, f_{6}, f_{8}, f_{10}, f_{32}, f_{34}, f_{36}, f_{38}\right\}, \\
\left\{f_{7}, f_{9}, f_{31}, f_{33}, f_{35}, f_{37}, f_{43}\right\}
\end{array}\right\}
$$

To synthesize the above analysis of coding and constraints, taking the length of the genetic code and the performance code is 40 , and selecting the population size is 40 , the original population is generated randomly, making selection uses the method of roulette selection, cross rate is 0.7 , mutation rate is 0.2 . After 300 generations of evolution using Matlab, we get the best individual:

$[7,9,1,4,31,33,43,11,23,16,13,15,12,21,14,17,20,8,10,2,5,35$, $37,32,34,44,27,19,25,28,30,26,22,18,27,34,21,29,24,39,41,4$ $0,42,36,38,3,6]$,

Where the times of clamping and changing knife are respectively 6 times and 8 times,and the result satisfies the clustering constraints, its fitness is 96.37 .

In view of this part, the process sequence manifested through genetic algorithm optimization is as follows: the rough milling of the front and back surfaces; drilling 1,2, the rough milling of the upper and lower surfaces, the rough milling of the lower slope, the rough milling of 10 cavities, the finish-milling of the front and back surfaces, reaming hole 1,2 , the rough milling of the left and right surfaces, the finish-milling of the upper and lower surfaces, the finshmilling of the lower slope, the finish-milling of 10 cavities, the rough milling of the keyway1,2, the finish-milling of the keyway 1,2 , the finish-milling of the left and right surfaces and milling the hole1,2.

Analyzing the result of optimization, the process sequence optimized by genetic algorithm is more reasonable.

\section{CONCLUSION}

In the CAPP system, solving the sorting problem of process sequence is always one of the difficulties. This paper uses genetic algorithm, in the corresponding constraints, by copying, crossover and mutation, to conduct a global optimization for the sorting of process route and finally uses an example to validate its good result. This study has important implications for the study of CAPP system process diagram's automatical generation.

\section{ACKNOWLEDGEMENTS}

The authors would like to thank the financial support from Scientific Research Innovation Fund of Shanghai University of Engineering Science and the school's lab conditions to this research. This paper comes from Project source: Graduate research and innovation projects of SUES . Project number: E1-0903-14-01005. 


\section{REFERENCES}

[1]. Joseph H. M. Tah. A Data Base Management System for Forwork Design Applications[J].Oxford Brookes University, 2008,5(1): 59-70

[2]. Shobrys D E. White D C. Planning, scheduling and control systems: why can they not work together[J]. Computer \& Chemical Engineering, 2000(24) : 163-173.

[3]. Bian Min. Discussion about the Type of CAPP System and Working Principle[J]. Technology Information, 2011, 01:15-16

[4]. F.Mervyn, A. Senthil kumar, S.H.Bok,A.Y.C.Nee, Development of an Internet-enabled 64 interactive fixture design system. Computer-Aided Design,2003,35: 945 957 [5]. B.K.Choi,K.Ko. C-space based CAPP algorithm for freeform die-cavity machining. Computer-Aided Design, 2003,35: 179 189

[6]. Renner.Gabor, Ekart.Aniko. Genetic algorithms in computer aided design. Cpmouter-Aided Design, 2003,

7:709 726

[7]. Chen,Yu-Ming. Management of water resources using improved genetic algorithms. Computers and Electronics in Agriculture, 1997, 8: 117 127

\section{BIOGRAPHIES}

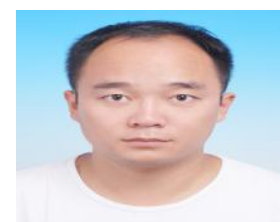

Shen Lei currently is a master in the Mechanical manufacturing and automation at Shanghai University of Engineering Science. His main research direction is application of CAD/CAM.

E-mail:shenleiswl3258@163.com

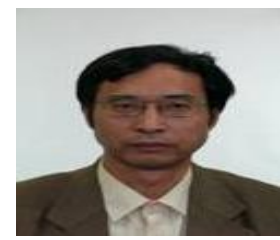

Yigang $\mathrm{Hu}$ currently is professor, working in Shanghai University of Engineering Science. His main research direction is technology and application of mechanical CAD/CAM

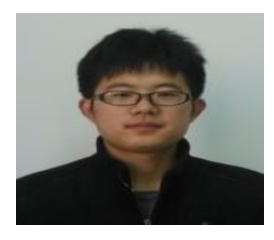

Cheng Jiang currently is a master in the Mechanical manufacturing and automation at Shanghai University of Engineering Science. His main research direction is automatic generation of process chart in CAPP system..

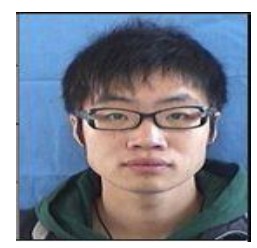

Liu Yuan currently is a master in the Mechanical manufacturing and automation at Shanghai University of Engineering Science. His main research direction is optimization of mechanical design. 